\title{
Trends in triathlon performance: Effects of sex and age
}

\author{
Lepers, Romuald ; Knechtle, Beat ; Stapley, Paul J
}

\begin{abstract}
The influences of sex and age upon endurance performance have previously been documented for both running and swimming. A number of recent studies have investigated how sex and age influence triathlon performance, a sport that combines three disciplines (swimming, cycling and running), with competitions commonly lasting between 2 (short distance: 1.5-km swim, 40-km cycle and 10-km run) and $8 \mathrm{~h}$ (Ironman distance: 3.8-km swim, 180-km cycle and 42-km run) for elite triathletes. Age and sex influences upon performance have also been investigated for ultra-triathlons, with distances corresponding to several Ironman distances and lasting several days, and for off-road triathlons combining swimming, mountain biking and trail running. Triathlon represents an intriguing alternative model for analysing the effects of age and sex upon endurance and ultra-endurance $(>6 \mathrm{~h}$ ) performance because sex differences and age-related declines in performance can be analysed in the same individuals across the three separate disciplines. The relative participation of both females and masters athletes (age $>40$ years) in triathlon has increased consistently over the past 25 years. Sex differences in triathlon performance are also known to differ between the modes of locomotion adopted (swimming, cycling or running) for both elite and non-elite triathletes. Generally, time differences between sexes in swimming have been shown to be smaller on average than during cycling and running. Both physiological and morphological factors contribute to explaining these findings. Performance density (i.e. the time difference between the winner and tenth-placed competitor) has progressively improved (time differences have decreased) for international races over the past two decades for both males and females, with performance density now very similar for both sexes. For agegroup triathletes, sex differences in total triathlon performance time increases with age. However, the possible difference in age-related changes in the physiological determinants of endurance and ultra-endurance performances between males and females needs further investigation. Non-physiological factors such as low rates of participation of older female triathletes may also contribute to the greater age-related decline in triathlon performance shown by females. Total triathlon performance has been shown to decrease in a curvilinear manner with advancing age. However, when triathlon performance is broken down into its three disciplines, there is a smaller age-related decline in cycling performance than in running and swimming performances. Age-associated changes in triathlon performance are also related to the total duration of triathlon races. The magnitude of the declines in cycling and running performances with advancing age for short triathlons are less pronounced than for longer Ironman-distance races. Triathlon distance is also important when considering how age affects the rate of the decline in performance. Off-road triathlon performances display greater decrements with age than road-based triathlons, suggesting that the type of discipline (road vs. mountain bike cycling and road vs. trail running) is an important factor in age-associated changes in triathlon performance. Finally, masters triathletes have shown relative improvements in their performances across the three triathlon disciplines and total triathlon event times during Ironman races over the past three decades. This raises an important issue as to whether older male and female triathletes have yet reached their performance limits during Ironman triathlons.
\end{abstract}

DOI: https://doi.org/10.1007/s40279-013-0067-4 
Journal Article

Accepted Version

Originally published at:

Lepers, Romuald; Knechtle, Beat; Stapley, Paul J (2013). Trends in triathlon performance: Effects of sex and age. Sports Medicine, 43(9):851-863.

DOI: https://doi.org/10.1007/s40279-013-0067-4 


\title{
Trends in triathlon performance: Effects of sex and age
}

\author{
Romuald Lepers ${ }^{1}$, Beat Knechtle ${ }^{2}$ and Paul J. Stapley ${ }^{3}$ \\ ${ }^{1}$ INSERM U1093, Faculty of Sport Sciences, University of Burgundy, Dijon, France \\ ${ }^{2}$ Institute of General Practice and Health Services Research, University of Zurich, Zurich, \\ Switzerland \\ ${ }^{3}$ School of Health Sciences, Neural Control of Movement Laboratory, Faculty of Medicine, \\ Science and Health, University of Wollongong, Australia
}

Word count: 6433

Key words: Endurance performance, Gender difference, Running, Cycling, Swimming, Aging, Masters athletes.

Running header: Sex, age and triathlon performance

\section{Corresponding author:}

Romuald Lepers

INSERM U1083

Université de Bourgogne

Faculty of Sport Science

BP 27877

21078 Dijon cedex, France

Tel : +33.3.80.39.67.60 / Fax : +33.3.80.39.67.02

Email : romuald.lepers@u-bourgogne.fr

\section{Acknowledgements}

This research project was not funded. All authors declare that there were no conflicts of interests. 


\section{Contents}

Abstract

1. Introduction

2. Sex differences in performance

2.1 Female rates of participation

2.2 Physiological and morphological considerations

2.3 Sex differences in triathlon performance

2.4 Sex differences in triathlon swimming performance

2.5 Sex differences in triathlon cycling performance

2.6 Sex differences in triathlon running performance

2.7 Performance density in triathlon results

2.8 Effects of age on sex differences

3. Age-related declines in triathlon performance

3.1 Effects of the mode of locomotion

3.2 Effects of discipline duration

3.3 Road-based versus off-road triathlon

3.4 Improvements in triathlon performance of elderly triathletes

4. Conclusion 


\section{Abstract}

The influences of sex and age upon endurance performance have previously been documented for both running and swimming. A number of recent studies have investigated how sex and age influence triathlon performance, a sport that combines three disciplines (swimming, cycling and running), with competitions commonly lasting between two (Short distance : 1.5 km swim, $40 \mathrm{~km}$ cycle and $10 \mathrm{~km}$ run) and eight hours (Ironman distance: $3.8 \mathrm{~km}$ swim, 180 $\mathrm{km}$ cycle and $42 \mathrm{~km}$ run) for elite triathletes. Age and sex influences upon performance have also been investigated for ultra-triathlons with distances corresponding to several Ironman distances and lasting several days, and for off-road triathlons combining swimming, mountain biking and trail running. Triathlon represents an intriguing alternative model for analyzing the effects of age and sex upon endurance and ultra-endurance (> 6 hours) performance because sex differences and age-related declines in performance can be analyzed in the same persons across the three separate disciplines.

The relative participation of both females and masters athletes (age > 40 years old) in triathlon has increased consistently over the past 25 years. Sex differences in triathlon performance are also known to differ between the modes of locomotion adopted (swimming, cycling or running) for both elite and non-elite triathletes. Generally, time differences between sexes in swimming have been shown to be smaller on average than during cycling and running. Both physiological and morphological factors contribute to explaining these findings.

Performance density (i.e. the time difference between the winner and $10^{\text {th }}$ placed competitor) has progressively improved (time differences have decreased) for international races over the past two decades for both males and females, with performance density now very similar for both sexes. For age-group triathletes, sex differences in total triathlon performance time 
increases with age. The possible difference in age-related changes in the physiological determinants of endurance and ultra-endurance performances between males and females needs further investigation however. Non-physiological factors such as low rates of participation of older female triathletes may also contribute to the greater age-related decline in triathlon performance shown by females.

Total triathlon performance has been shown to decrease in a curvilinear manner with advancing age. However, when triathlon performance is broken down into its three disciplines, there is a smaller age-related decline in cycling performance compared to running and swimming performances. Age-associated changes in triathlon performance are also related to the total duration of triathlon races. The magnitude of the declines in cycling and running performances with advancing age for short triathlons are less pronounced than for longer Ironman distance races. Triathlon distance is also important when considering how age affects the rate of the decline in performance. Off-road triathlon performances display greater decrements with age than road-based triathlons suggesting that the type of discipline (road versus mountain bike cycling and road versus trail running) is an important factor in ageassociated changes in triathlon performance. Finally, masters triathletes have shown relative improvements in their performances across the three triathlon disciplines and total triathlon event times during Ironman races over the past three decades. This raises an important issue as to whether older male and female triathletes have yet reached their performance limits during Ironman triathlons. 


\section{Introduction}

Triathlon is a unique endurance sport that combines three disciplines (swimming, cycling and running) over a variety of distances. ${ }^{[1]}$ Triathlon first appeared at the end of 1970's and in only one-quarter of a century has developed into a well-organized sport with global participation. The first Hawaii Ironman triathlon consisting of a $3.8 \mathrm{~km}$ swim, $180 \mathrm{~km}$ cycle and $42 \mathrm{~km}$ run had 12 male participants in 1978. Currently, more than 1700 triathletes $(<30 \%$ females), most of whom have to qualify at one of 25 Ironman triathlons worldwide, participate in this event which has become the Ironman World Championship. ${ }^{[2]}$ Short or Olympic distance triathlons $(1.5 \mathrm{~km}$ swim, $40 \mathrm{~km}$ cycle and $10 \mathrm{~km}$ run) have also rapidly grown in popularity during approximately the same period with the first World Championship being held in Avignon (France) in 1989. Triathlon was officially accepted into the Olympic games as a full sport in Sydney in 2000. ${ }^{[3]}$ Ultra-triathlons, consisting of distances greater than Ironman distance include double Ironman triathlons (7.6km swim, $360 \mathrm{~km}$ cycle and $84 \mathrm{~km}$ run) and decaIronman triathlon $(38 \mathrm{~km}$ swim, $1800 \mathrm{~km}$ cycle and $420 \mathrm{~km}$ run) also appeared during the 1980's with the first double Ironman triathlon held in 1985 in Huntsville (USA). ${ }^{[4,5,6,7]}$ More recently, in addition to conventional road based triathlon, off-road triathlon combining swimming, mountain biking and trail running has also established itself in the field of endurance sports, and has grown very rapidly in popularity. ${ }^{[8,9]}$

Triathlon performance has been studied from numerous perspectives by a number of research groups over approximately the same period that the sport has grown. Research has investigated physiological $^{[1,10,11,12,13,14]}$, biomechanical ${ }^{[15,16,17]}$, training ${ }^{[16,18,19,20]}$, nutritional ${ }^{[21]}$ or medical ${ }^{[22]}$ aspects of triathlon performance. Although Ironman triathlon is a relatively new ultra-endurance event, recently elite Ironman triathletes appear to have reached their performance limits ${ }^{[2,23]}$ as has been observed in more traditional sports such as marathon 
running where performance times have plateaued. ${ }^{[24,25]}$ With the increase in popularity of endurance sports worldwide, endurance sports have attract a greater participation of female and masters athletes (age $>40$ years) during recent times. ${ }^{[24,25,26,27]}$

This review addresses the specific aspects of: i) sex differences and ii) age-related declines in triathlon performance. Sex differences in performance will be presented and discussed for each of the three disciplines and total event times for different distances and for both elite and age group triathletes. Age-related declines will be outlined by focusing on the performances of the best master triathletes because these athletes represent a unique model for studying the effects of high levels of physical training in persons of older age.

\section{Sex differences in performance}

\subsection{Female rates of participation}

Independent of age, the number of females competing in triathlon has increased progressively since the 1980's. For example, between 2000 and 2011, female USA Triathlon membership has grown from $27 \%$ of the total number of annual members to more than $38 \% .{ }^{[28]}$ Factors leading to this growth include society's increasing acceptance of "active" females; females feeling more comfortable living an active lifestyle ${ }^{[29]}$, and the growth of females-only events, good examples of which are the "Danskin" and "Trek Triathlon" Series in USA.

The rates of female participation appear to decrease with an increase in distance of triathlon races. For example, in 2010 females accounted for $26 \%, 19 \%$ and $13 \%$ of athletes competing at short, half-Ironman and full-Ironman distance triathlons held in the 
region of Zurich (Switzerland) ${ }^{[30,31,32]}$. However, for ultra-triathlons, female participation remains relatively low, representing generally less than $10 \%$ of overall participant numbers. ${ }^{[4,5,33]}$

The number of females finishing the Hawaii Ironman World Championship increased from 20 in 1981 (6\% of participants) to more than 470 in 2010 (27\% of participants). ${ }^{[34]}$ In Europe, there was also a progressive rise in the number of female Ironman finishers but overall rates were lower. In 2011, females accounted for $13 \%$ of the field at Ironman Switzerland. ${ }^{[32]}$ Lower rates of female participation in Europe may because European Ironman triathlon is relatively younger compared to the Hawaii Ironman triathlon. Indeed, the first Hawaii Ironman was held in 1978, while one the first European Ironman, Ironman Switzerland, was held in 1995. Female participation in Ironman triathlon remains slightly lower compared to traditional endurance events such as marathon running, but greater than ultra-endurance events such as a 161-km ultramarathon. For example, Lepers \& Cattagni ${ }^{[24]}$ showed that the relative participation of females at the New-York City Marathon increased over the last 30 years from $17 \%$ to $33 \%$ of the total field. In contrast, females accounted for $20 \%$ of the finishers in the same time period at 161-km ultramarathons in North America. ${ }^{[35]}$

\subsection{Physiological and morphological considerations}

Physiological and morphological characteristics may account for the sex differences seen in triathlon performance. Until the 1970's, data was scarce with regard to the physiological determinants of female in endurance sports. Even though there is a lesser amount of data for females compared to males, studies have shown that maximal 
oxygen uptake ( $\dot{\mathrm{V}} \mathrm{O}_{2 \max }$ ), lactate threshold and running economy interact similarly in females as determinants of endurance performance as they do in males. ${ }^{[36]}$

Current explanations for sex differences in $\dot{\mathrm{V}} \mathrm{O}_{2 \max }$ among elite athletes, when expressed relative to body mass, provide two major findings. ${ }^{[37,38]}$ First, elite females have more $(<13 \%$ vs. $<5 \%)$ body fat than males. ${ }^{[39]}$ Indeed, much of the difference in $\dot{\mathrm{V}} \mathrm{O}_{2 \max }$ between males and females disappears when it is expressed relative to lean body mass. ${ }^{[37,38]}$ Second, the hemoglobin concentration of elite athletes is $5-10 \%$ lower in females than in males. ${ }^{[40]}$ Concerning lactate threshold, there is no reason to believe that values should be lower in females than in males because mitochondrial adaptations in the skeletal muscles of highly-trained male and female athletes appear to be similar. ${ }^{[41]}$ Finally, the average oxygen cost to run a given speed, (i.e. running economy) by groups of elite male and female athletes is similar and appears to play the same role in determining success in endurance performance. ${ }^{[42]}$ Therefore, the major physiological reason that explains the slower record performances by females compared to males is probably the lower $\dot{\mathrm{V}} \mathrm{O}_{2 \max }$ values observed in females.

Physiological differences between male and female triathletes have been few investigated. ${ }^{[14,39,43]}$ The differences in the physiological responses in cycling and the energy cost of running after cycling have been compared in males and females for both elite junior and senior triathletes. ${ }^{[14]}$ These authors showed that senior females possessed a significantly higher cycling peak power output than their junior counterparts. Additionally, senior males had a higher ventilatory threshold than junior males whereas the ventilatory threshold was similar in junior and senior females.

A recent study conducted on junior triathletes has suggested that morphological characteristics of triathletes have evolved since late 1990's. ${ }^{[44]}$ In 2011 , both male and 
female junior elite triathletes appeared more ectomorphic than their 1997 counterparts. Nowadays, junior triathletes are proportionally lighter, with significantly smaller flexed arm and thigh girths, and femur breadths. The junior males also have significantly longer segmental lengths and lower endomorphy values than the 1997 junior males. More research is required however, to validate these findings for senior triathletes.

Male triathletes possess a larger muscle mass, greater muscular strength and lower relative body fat compared to female triathletes. ${ }^{[45]}$ Low body fat is an important predictor variable for total time performance in triathlon. For example, Knechtle et al. ${ }^{[46]}$ showed that low body fat was associated with faster race times in male Ironman triathletes but not in females. Males possess on average 7-9\% less percent body fat than females, which is likely an advantage for males. Therefore, it appears that sex differences in percentage body fat, oxygen carrying capacity and muscle mass may be major factors for sex differences in overall triathlon performance. Menstrual cycle, and possibly pregnancy, may also impact training and racing in female athletes, factors that do not affect males. ${ }^{[4]}$

\subsection{Sex differences in triathlon performance}

Sex differences in endurance and ultra-endurance $(>6 \mathrm{~h})^{[48]}$ performance have received considerable attention over the past few decades, but the majority of studies have focused on running performances. ${ }^{[49,50]}$ Interestingly, Speechly et al. ${ }^{[51]}$ reported that females, who were matched with males for a $42-\mathrm{km}$ run, were faster than males in a $90-\mathrm{km}$ race. Similarly, it has been shown that females and males who were matched for 50-km trail running performance also performed similarly in trail runs of 80 - and 161-km distances. ${ }^{[52]}$ Despite the suggestion in 1992 that females may one day outrun males in competitive ultra-endurance events ${ }^{[53]}$, elite males appear to run approximately $10-12 \%$ faster than elite females across all endurance 
running race distances up to marathon, with the sex difference narrowing as the race distance increases. ${ }^{[49,50]}$ However, at distances greater than $100 \mathrm{~km}$, such as the $161-\mathrm{km}$ ultramarathon, the difference seems even larger, with females $20-30 \%$ slower than males. ${ }^{[49,54,55]}$

\section{Insert TABLE 1}

Sex differences in triathlon performance have previously been described for elite triathletes ${ }^{[2,9,31,56,57]}$ and for non-elite triathletes of different age groups ${ }^{[30,34,58]}$ for long distances (distances greater or equal to half-Ironman) ${ }^{[2,31,34,56,57]}$ and for off-road triathlon $^{[9]}$. Surprisingly, very little data is available for short distance triathlons especially for high-level international races. One reason may be that the top international short distance triathlons, (i.e. World Championship Series events or Olympics) have all been draft legal for several years and therefore it is difficult to find a reference of high level Olympic distance triathlon without drafting for comparison. The increase in nondrafting international distance triathlons such the $H y$-Vee triathlon that offers significant prize money of $\$ 151,000$ USD for both male and female winners and represents a highly competitive platform for professional triathletes should help to better determine sex differences in short distance triathlon performance when athletes compete on a level (non-drafting) playing field in the future.

Table 1 synthesizes the data from the literature that has focused on sex differences in triathlon performances including international and national triathlon races, from age-groups and elite triathletes. Over the past 25 years, sex differences in total triathlon performance (across triathlons of different distances and format) have varied from $12 \%$ to $18.2 \%$ (far right column, Table 1), depending on the level of the triathletes (elite versus age group) or the distance/format of the race. Overall however, data has shown that the sex difference in triathlon performance has narrowed across the years. ${ }^{[2]}$ Table 2 presents most recent data of 
sex differences in triathlon performance for international elite triathletes at the most competitive races for each triathlon distance. By 2012, values narrowed so much that, for the top 10 triathletes overall in three top level international road-based triathlon races, the difference between males and females was $11.3 \%$ for the Hawaii Ironman triathlon, $14.1 \%$ for the Olympics and $9.3 \%$ for the Hy-Vee short distance triathlon (see Table 2). Non road-based triathlon produced a greater sex difference (discussed in more detail below). Interestingly, for ultra-triathlons, it has been shown that with increasing length of the event, the best females became relatively slower compared to the best males. ${ }^{[4]}$ Indeed, if the world's best performances are considered, males were $19 \%$ faster than the females in both Double and Triple Ironman distance, and $30 \%$ faster in the Deca-Ironman distance. ${ }^{[4]}$ However, nonphysiological factors may have contributed to these observations, in particular a fewer overall number of female finishers in ultra-triathlons, compared to formats of more standard length. ${ }^{[4]}$

\subsection{Sex differences in triathlon swimming performance}

The average sex difference in swimming performance during triathlon for race distances between 1.5 and $3.8 \mathrm{~km}$ ranged between approximately $10 \%$ and $15 \%$ for elite triathletes (see Table 1). The sex difference in triathlon swimming performance is consistent with values found for different pool swimming events. It has been shown that sex differences in pool swimming performances become progressively smaller with increasing distance from $50 \mathrm{~m}(19 \%)$ to $1500 \mathrm{~m}(11 \%) .{ }^{[59]}$ This seems also to be the case for triathlon as Table 1 shows that, the longer the distance of the swim component of a triathlon race, the smaller the sex difference, particularly when the top 10 age group athlete performances are considered. In ultra-endurance outdoor swimming events, female swimmers have tended to reduce the gap with their male counterparts. For 
example, time differences between male and female swimming records are $6.7 \%$ for the 32-km "English Channel Swim" and 2.3\% for the 26-km "Marathon Swim in Lake of Zurich" in Switzerland, respectively. ${ }^{[60,61]}$ However, the sex difference in performance between the best male and female ultra-swimmers is more generally close to $11-12 \%$, which corresponds to values observed for swimming in triathlon. ${ }^{[60,61,62]}$ It has been shown that the elite male and female triathletes completed the $3.8-\mathrm{km}$ swim stage of the Hawaii Ironman triathlon $\sim 10 \%$ slower than the elite swimmer specialists for the same distance at the Waikiki Roughwater Swim race. ${ }^{[2]}$ This difference could be explained by better propelling efficiency in elite swimmers compared with elite triathletes. ${ }^{\text {[63] }}$

Analysis from 2005 showed that the sex difference in triathlon swimming appears lower than in cycling and running at the Hawaii Ironman triathlon and at the off-road triathlon Xterra World Championship. ${ }^{[2,9,34]}$ The difference between swimming and the two other disciplines could be explained in part by the biological difference in relative body fat (7-9 \% higher in females). ${ }^{[64,65]}$ Performance in activities that involve supporting ones weight, for example running, may be more susceptible to a greater body fat percentage compared to water-based events such as swimming, during which body fat can increase buoyancy and therefore improve performance. ${ }^{[65]}$ Buoyancy is also improved in females through a lower 'underwater torque', which can be defined loosely as the tendency for the feet to sink. ${ }^{[66]}$ In addition, in contrast to running where the energy cost appears to be similar between females and males, the energy cost of freestyle swimming has been shown to be significantly higher (i.e. lower economy) in males compared to females. ${ }^{[66,67]}$ At a velocity of $1 \mathrm{~m} / \mathrm{s}$, there are differences in drag force and coefficient of drag between males and females. ${ }^{[68]}$ The energy cost of swimming depends essentially on the propelling efficiency of the arm stroke and hydrodynamic resistance, but it has been suggested that differences in energy costs of swimming between sexes are 
mainly to be attributed to differences in hydrodynamic resistance. ${ }^{[69]}$ In contrast, differences in energy cost of swimming across ages may be attributed also to changes in the propelling efficiency of the arm stroke. ${ }^{[69]}$ Females have smaller body size (resulting in smaller body drag), smaller body density (greater fat percent) and shorter lower limbs, resulting in a more horizontal and streamlined position and therefore a smaller underwater torque. ${ }^{[64,66]}$

\subsection{Sex difference in triathlon cycling performance}

Sex differences in triathlon cycling vary from 12 to $16 \%$ according to the level of expertise of participating triathletes for road-based triathlons (Table 1). Data gained from analysing male and female participants' performances in the $180-\mathrm{km}$ cycling leg of an Ironman triathlon are unique datasets as official time-trial road-cycling championships generally take place on distances much shorter than $180-\mathrm{km}$, with distances also being greater for males $(<40 \mathrm{~km})$ than for females $(<25 \mathrm{~km})$. Moreover, there is a paucity of data concerning sex differences in road or track cycling performance. ${ }^{[70,71,72]}$ In track cycling, where females are generally weaker than males in terms of power/weight ratios, the performance gap between males and females appears to be constant $(<11 \%)$ and independent

of the race distance from 200 to $1000 \mathrm{~m} \cdot{ }^{[70]}$ In ultra-cycling events, such as the "Race Across America", sex difference in performance was around $15 \%$ among top competitors. ${ }^{[71]}$ Greater muscle mass and aerobic capacity in males, even expressed relative to the lean body mass ${ }^{[73]}$, may represent an advantage during long distance cycling, especially on a relative flat course such as Ironman cycling where cycling approximates to a non-weight-bearing sport. Indeed, it has been shown that absolute power output (which is greater for males than for females) is 
associated with successful cycling endurance performance because the primary force inhibiting forward motion on a flat course is air resistance. ${ }^{[74]}$

Interestingly, for elite triathletes the sex difference in mountain bike cycling during off-road triathlon $(<20 \%)$ is greater compared to cycling sex differences in conventional roadbased events. ${ }^{[9]}$ Mountain biking differs in many ways from road cycling. Factors other than aerobic power and capacity such as off-road cycling economy, anaerobic power and capacity, and technical ability might influence off-road cycling performance. ${ }^{[75]}$ Bouts of high intensity exercise frequently encountered during the mountain biking leg of off-road triathlon (lasting < 1h 30 min for elite males and $<2 \mathrm{~h}$ for elite females) can result from: 1) having to overcome the constraints of gravity associated with steep climbs, 2) variable terrain necessitating wider tires and thus greater rolling resistance, and 3) isometric muscle contractions associated with the needs of more skilled bike handling skills, not so often encountered in road cycling. In particular however, lower power-to-weight ratios for females compared to male triathletes inevitably leave them at a disadvantage during steep climbs. ${ }^{[76,77]}$ Moreover, the increased bike handling skills, required especially during downhill mountain biking may be impaired by relatively lower female arm or leg isometric muscle strength, meaning that greater sex differences in off-road triathlon could be attributed to decreased ability to control the bicycle in females. However, this assumption needs to be confirmed with specific investigations of the effect of sex on technical ability in mountain biking. ${ }^{[9]}$

As percentage differences in time do not equate to percent differences in power output, due to non-linear relationships between speed and power output from air or water resistance $^{[78]}$, the magnitude of the sex difference has also been examined by calculating the percentage difference between males and females in estimated power outputs for each discipline. ${ }^{[2,9,34,79]}$ In this case, differences in estimated power output between the sexes are 
greater for triathlon cycling than for the swimming and running disciplines. For example, sex differences in power output in swimming, cycling and running were estimated to be $28 \%$, $39 \%$ and $33 \%$, respectively for Ironman triathlon, and 30\%, 45\% and 33\% for off-road triathlon. ${ }^{[9]}$ Because power output is proportional to oxygen uptake, the magnitude of sex differences in power output provides a more realistic representation of underlying sex differences in physiological capacity. ${ }^{[80]}$

\subsection{Sex differences in triathlon running performance}

During the 1988-2007 period, the top 10 elite males have run the Hawaii Ironman marathon on average $13.3 \%$ faster than the top 10 females. ${ }^{[2]}$ In contrast, during the same period, elite female triathletes have improved their marathon running times by $0.8 \mathrm{~min} / \mathrm{yr}$ while times have remained stable for the males. While these improvements in female performances compared to males are impressive, they remain as yet unexplained, which is strange when it is considered that females have benefited from similar training and nutritional advances. ${ }^{[21]}$ If females continue to improve their running performance at Ironman, they could reduce the sex difference in the marathon leg and therefore their overall performance times. For example, at the 2012 Hawaii Ironman, the difference in marathon times between the top 10 elite males and the top 10 elite females reduced to $7.3 \%$ (males: $<3 \mathrm{~h} 05 \mathrm{~min}$, females: $<3 \mathrm{~h}$ 20min) (Table 2). Another notable example is that the female winner of the famous Roth Ironman (Germany) in 2011 ran only $2.6 \%$ slower than the winning male ( $2 \mathrm{~h} 44 \mathrm{~min}$ vs. $2 \mathrm{~h}$ 40min). At the same race, both female and male winners bettered the Ironman distance performance world record ( $8 \mathrm{~h} 18 \mathrm{~min}$ vs. $7 \mathrm{~h} 41 \mathrm{~min}) .{ }^{[81]}$ Thus, it appears that the marathon running leg of Ironman triathlon has become the discipline in which female elite triathletes have most reduced the gap with their male counterparts. Interestingly, Lepers ${ }^{[2]}$ showed that 
for the top 10 elite finishers the sex difference in running at the Hawaii Ironman marathon was similar to that recorded during the New-York marathon, suggesting above all, that the swim and cycle legs of the triathlon do not exacerbate the sex difference in running a marathon. Thus, the physiological differences between males and females in running performance that have been identified to occur in a regular marathon still persist in the marathon of an Ironman.

Contrary to the knowledge of running performance differences between sexes for the marathon distance, few analyses have been conducted between males and females who have run 10-km during an Olympic distance triathlon. A mean sex difference of 17\% was found for the Zurich Switzerland Olympic distance triathlon during the $2000-2010$ period ${ }^{[30]}$ However, as Table 2 shows, when data from 2012 only is considered, there is a considerable difference even within elite level Olympic distance run performances, between males and females. A difference of $12.3 \%$ existed during the draft-legal Olympic short distance race in London, whereas the difference was lower (8.6\%) at the Hy-Vee international triathlon in Des Moines (draft-free). It would appear that the format of the triathlon (drafting or not) affects males differently to females, with the possibility that males benefit in terms of their subsequent running performance off the bike, to a greater extent when riding in a peloton. However, running performances in males and females need to be more closely analyzed in relation to the effort produced during the cycle leg of an Olympic distance triathlon.

\section{Insert TABLE 2}

\subsection{Performance density in triathlon results}

Performance density (i.e. the time difference between the winner and $10^{\text {th }}$ placed competitor) has been quantified by considering overall performance times in triathlon. ${ }^{[2]}$ 
For example, at the Hawaii Ironman triathlon between 1981 and 2008, the average time difference between 1 st and $10^{\text {th }}$ place was smaller for male $(5.8 \%)$ than for female athletes $(7.5 \%) .{ }^{[2]}$ During the past five years, the performance density has decreased for both male $(<3.1 \%)$ and female athletes $(<5.7 \%)$, a trend which would suggest that in the future, high performing female athletes may be as performance dense as the males. In fact, Table 2 indicates that in 2012 performance density was similar across the two sexes for top international road-based triathlon events, regardless of format (Hawaii, Olympics and $\mathrm{Hy}$-Vee). A notable exception to this was the Xterra World Championships (off-road triathlon) in which female performance density (12.2\%) was considerably lower than the male performance density (4.6\%). As explained earlier (section 2.5), this can likely be explained by the specific physiological and technical abilities needed for the mountain bike leg of Xterra, in particular explosive strength which favours males over females. ${ }^{[82]}$ The small performance density observed for the London Olympics compared to other events, for both males and females, may be explained by the drafting component of the cycling leg that led to a grouped start of a number of athletes (approximately half of the field) at the beginning of the run leg. It must also be considered that a number of nations fielded $>1$ athlete, and identified a designated leader who was 'protected' during the swim/cycle legs. This would also have led to a higher overall athlete density in terms of overall performance times in both males and females.

\subsection{Effects of age on sex differences}

Physiological, (e.g. lower muscle strength and oxygen carrying capacity), morphological, (e.g. greater percentage of body fat, lower muscle mass), and functional capacities are well known to change with advancing age in both males and females. ${ }^{[83]}$ In addition, is has been shown that, after 55 years of age, the decline in endurance 
performance is more pronounced in females than in males. ${ }^{[84]}$ It is thus likely that sex differences in triathlon performance become more pronounced with advancing age. A greater age-related decline in performance in females compared to males has been previously observed in swimming and running. ${ }^{[59,85]}$ Results from the Hawaii Ironman triathlon showed that the sex difference in total event performance time increased significantly with advancing age from 55 yrs during the 2006-2008 period. ${ }^{[34]}$ Sixty year old male triathletes were on average $27 \%$ slower than those that were between 30 and 40 years of age, while the difference was $38 \%$ for females. Sex differences in performance during a Swiss regional Olympic distance triathlon became greater over the age of 35 years. ${ }^{[30]}$ The occurrence of this large sex difference was somewhat earlier with respect to athletes' age than recorded during the Hawaii Ironman triathlon. ${ }^{[34]}$ Possible explanations for this include the greater competitive level of older participants at the Hawaii Ironman triathlon because athletes are required to qualify as it is a World Championship event. There is a need for further research to understand if exercise duration exerts an influence upon sex differences across older age groups by analysing performances of athletes in a wider range of triathlon events, from Sprint distance, through Olympic distance and Halfironman, to Ironman distances. ${ }^{[79]}$

The exact reasons for these sex-related differences with advancing age are currently not clear but may result from physiological, sociological and psychological changes. ${ }^{[38,86,87]}$ For example, a greater decline of one or more physiological determinants of endurance performance for females compared to males, (e.g. $\dot{\mathrm{V}} \mathrm{O}_{2 \max }$, lactate threshold, or exercise economy), or a difference in age-related changes in body composition (increase in percentage body fat and loss of muscular mass), hormonal changes, and fluid balance changes (e.g. decline in the thirst mechanism), could affect triathlon performance. ${ }^{[38,83,84,88]}$ In addition, differences in terms of years of training, training 
volume and intensity between elderly male and female triathletes performing Ironman triathlon may exist, but further research is needed to clarify this. However, interpretation of cross-sectional comparisons of triathlon performance times across ages and sexes must be made carefully. It is likely that, compared to males, there are fewer females competing in triathlon events, especially in the older age categories. For example, the percentage of females participating at the Hawaii Ironman triathlon during 2006-2008 corresponded on average to $27 \%$, but "finisher" females in the age group 60-64 yrs represented only $3 \%$ of the females field. ${ }^{[34]}$ This participation difference may diminish over the next couple of decades as has been observed in marathon running ${ }^{[24,26]}$, such that because the welltrained females move up to the older age groups, the improvement of the oldest females may actually surpass the oldest males.

\section{Age-related declines in triathlon performance}

Age-related declines in endurance and ultra-endurance performance have been well described in the literature for running ${ }^{[24,35,38,54,83,89,90,91]}$, cycling ${ }^{[92,93,94,95]}$, swimming ${ }^{[83,85]}$ and more recently, for triathlon. ${ }^{[34,79,92,96,97,98]}$ Endurance and ultra-endurance performance appears to be maintained until approximately 35-40 years of age, followed by modest decreases until 50 years of age and a progressive decrease in performance thereafter. ${ }^{[83,84,88]}$ The greatest declines in endurance and triathlon performance occur after the age of 70 years (Table 3). Physiological factors contribute to age-related declines in endurance performance in older athletes and there is obviously an interaction between training behaviour and performance in older athletes. ${ }^{[38,83,86,88]}$

It has been found that the age of peak performance in Ironman triathlon is around 3334 years for both males and females ${ }^{[32,57]}$, which seems to be older than the age of peak 
overall performance of marathoners. ${ }^{[99]}$ However, this finding observed for the Ironman Switzerland triathlon needs be confirmed for other Ironman events. For non-elite triathletes, the fastest race times are usually achieved between 25 and 44 years for both Ironman and Ultra-triathlons. $^{[32,57,97]}$

\subsection{Effects of the mode of locomotion}

Total triathlon performance decreases progressively in a curvilinear manner with advancing age. However, there is a smaller age-related decline in cycling performance compared to running and swimming performances for both short and long distance triathlons. $^{[8,96,97]}$ These findings suggest that age-related declines in endurance performance are specific to the mode of locomotion, although the cause for such modespecificity is not clear. Where does the focus of this locomotor mode-specificity with age lie? Our group has previously proposed that mechanical power could explain these agerelated differences in cycling and running. ${ }^{[98]}$ According to the formula $\mathrm{P}=\mathrm{k} . \mathrm{V}(\mathrm{k}$ : constant), mechanical power output (P) is dependent upon velocity (V) during running, whereas it is dependent upon the third power of velocity during cycling $\left(\mathrm{P}=\mathrm{k} \cdot \mathrm{V}^{3}\right)$. Following this, we previously suggested that as changes in aerobic capacity with age are tied to reductions in $\mathrm{P}$, those reductions during running and cycling with age would give rise to lower cycling velocities than running velocities. ${ }^{[98]}$ A number of explanations can be given to explain the smaller decline in cycling performance during triathlon compared to the declines in the other two disciplines. These include a lesser reduction in lactate threshold or economy during cycling, or a greater muscular fatigue during running with age, although these propositions remain to be validated. Some authors have though, attempted to explain this age-related phenomenon in cycling by proposing that the 
'training stimulus', the ability or will to train, is reduced in running compared to cycling. ${ }^{[85]}$ In particular, this may be because running is associated with a greater amount of orthopedic injuries, which limits the ability to train in running. ${ }^{[100]}$ The training stimulus would therefore be maintained in cycling due to the less traumatic nature of the endurance activity and therefore with age, triathletes would tend to cycle more than run. However, this proposition remains speculative and requires further investigation.

\subsection{Effects of discipline duration}

The duration of a triathlon race exerts an important influence on the age-related changes in triathlon performance. ${ }^{[98]}$ Age-related declines in swimming performance are not influenced by triathlon duration with the magnitudes of decreases in swimming performance similar for Olympic distance versus Ironman triathlon. However, in contrast to swimming the magnitude of the declines in cycling and running performances with advancing age during Olympic distance triathlon were less pronounced than during Ironman. ${ }^{[98]}$ For age group competitors of 70-74 yrs, total finishing time is approximately three hours for the Olympic distance triathlon whereas it is around 15 hours for an Ironman (Table 3). Certainly the Ironman triathlon induces greater neuromuscular fatigue in cycling and running compared to the Olympic distance event. ${ }^{[101]}$ Furthermore, muscle damage during a 10-k run of the Olympic distance triathlon is limited compared to that which occurs during an Ironman marathon. Greater muscle fatigability and greater sensibility to muscle damage of older triathletes needs further investigation to determine if these factors underlie the greater declines in cycling and running performance of older triathletes.

\subsection{Road-based versus off-road triathlon}


It has recently been shown that the rate of the decline in performance for off-road triathlon is greater than for road-based triathlon. ${ }^{[8]}$ This suggests that the type of discipline (road versus mountain bike cycling and road versus trail running) exerts an important influence on the magnitude of the age-associated changes in triathlon performance. ${ }^{[8]}$ The specific aspects of mountain biking and trail running may explain why age-related declines in off-road triathlon are more pronounced for off-road compared to conventional on road triathlon. In particular, a decrease in power-to-weight ratio that has been shown to occur with age $^{[102,103]}$ leaves older athletes with a distinct advantage during steep climbs often encountered in off-road triathlons. Moreover, despite a preservation of muscle strength in comparison to sedentary persons ${ }^{[104]}$, older athletes may be disadvantaged in their technical bike handling skills due to lower arm/leg muscle strength. Poorer running performances in older athletes, especially when variations in intensity are required, may be explained be a reduced ability to modify the biomechanical components of running (e.g., support phases, stride frequency, changes in stride length, etc. $)^{[8]}$

\section{Insert TABLE 3}

\subsection{Improvements in triathlon performance of elderly triathletes}

An increase in participation of both male and female athletes older than 40 yrs over the past few decades has been reported for marathon and ultra-marathon running, such as 161-km ultra-marathons and for $100-\mathrm{km}$ running in Switzerland. ${ }^{[24,25,35,90]}$ A relative increase in participation of masters triathletes has also been observed during the past decade for short and long distance triathlon, while the participation of triathletes younger than $40 \mathrm{yrs}$ of age has decreased. ${ }^{[27,28,30,58]}$ The relative increase in participation of master triathletes at the Ironman 
distance triathlons has been accompanied by an improvement in their performance. ${ }^{[27,105]} \mathrm{We}$ have previously presented data for the 1986-2010 period showing that swimming, cycling, running and total time performances at the Hawaii Ironman Triathlon improved for male triathletes older than 44 years age and female triathletes older than 40 years age. ${ }^{[27]}$ During the 25 -year study period, the total time decreased by $<21 \%$ (from $14 \mathrm{~h} 18 \mathrm{~min}$ to $11 \mathrm{~h} 16 \mathrm{~min}$ ) for the best male finishers in the age group 60 to 64 years and by the same percentage (from $14 \mathrm{~h}$ $38 \mathrm{~min}$ to $11 \mathrm{~h} 30 \mathrm{~min}$ ) for the best finishers in the age group 40 to 44 years. ${ }^{[27]}$ Similar findings have been observed for a Hawaii Ironman qualifying race such as the Switzerland Ironman. ${ }^{[105]}$ In Table 3, we show that, when the most recent data (Ironman Hawaii, 2011 and 2012) is added, improvements of older age groups, especially for the males has continued, and for some age groups, is quite startling. For example, the best male finisher in the 70-74 age group has bettered the best time set in 2010 (12h $41 \mathrm{~min}$ ) by almost one hour (11h $45 \mathrm{~min}$ ). Other, more modest improvements have been recorded in the male 65-69 and >80 age groups, and the female 45-49 age group with respect to the 2010 data. ${ }^{[27]}$ What is clear however, is that, if the most recent (2012) data is compared to that of 1986, age groups show improvements of between 114\% and $194 \%$ for the males over 45 years, and $106 \%$ and $179 \%$ for females over 45, and that these improvements seem to be continuing, most significantly for the oldest athletes.

Several reasons may explain the improved performance of master triathletes. These include an improvement of training facilities, coaching, training techniques, nutritional strategies and equipment, ${ }^{[84,86]}$ as well as the possibility that they have had better and prolonged access to the facilities required to train effectively. It is also obvious that the higher participation rates of master athletes increases the possibility of them obtaining better results due to the competitive nature of the sport. Additionally, athletes that have attained a number of good results and positive outcomes, have increased levels of motivation to train and compete in 
endurance events. ${ }^{[106]}$ Accordingly, the better physical condition of older athletes is likely to increase competitive spirit, participation and performance. ${ }^{[84,86]}$ The advancement of training quality is also a possible reason for these improved performance trends. Earlier studies suggested the performance decline with advancing age was due to decreased training volumes and intensity. ${ }^{[86]}$ Other, more sociological, factors may also play a role in reducing the ability and motivation to train. These include greater work demands, demands of the family, a diminished inherent drive to push oneself or even train. ${ }^{[83,84,86]}$ An important factor is also the greater amount of time needed by the body to recover from hard, physical effort resulting from training as humans age. Indeed, training for an Ironman triathlon is very demanding and in order to place in the top ten of an age group category, extremely high training volumes and intensities are required. ${ }^{[18,107,108,109]}$ However, it has been suggested that masters athletes could optimize their quality of training, so they could reduce their training volume to save time for adequate recovery and remain injury free. ${ }^{[84]}$ These studies suggest that masters triathletes have probably not reached their limits in ultra-endurance performance. Unfortunately, no information is available concerning the sporting background of these successful masters triathletes. Nevertheless, even if recreational masters athletes had relatively short training histories and little experience in triathlon, these successful athletes probably have lifelong histories of physical activity. ${ }^{[110,111,112,113]}$

\section{Conclusions}

Sex differences in triathlon performance differ generally between the locomotion modes with lower differences seen in swimming compared to cycling and running for both elite and non-elite triathletes. In elite triathletes, sex differences in performance $(\sim 10-14 \%)$ are in agreement with values generally observed in endurance sports. At present, the 
performance density in highly competitive international triathlon races appears to have become similar between elite male and female triathletes. Marathon running during an Ironman triathlon appears to be the discipline where female elite triathletes have reduced the gap with the males to the greatest extent over the recent years. Sex-related differences increase with advancing age most likely due to physiological, sociological and psychological changes. However, these differences should decrease in the future with the increase in participation of elderly female triathletes.

Age-related declines in triathlon performance depend on the locomotion mode, the exercise duration (short versus long distance triathlon) and the triathlon format (off-road versus road based triathlon). The participation of older triathletes has increased over the past 25 years and will probably continue to grow in the future. This increase in participation has been accompanied by an improvement in masters triathlon performance. The question whether older triathletes have yet reached limits in their performance should therefore be examined in more detail. Further studies investigating training regimes, competition experience or sociodemographic factors are needed to gain better insights into the phenomenon of the relative improvements in endurance and ultra-endurance performance with advancing age. 


\section{References}

1. Bentley DJ, Millet GP, Vleck VE, MacNaughton LR. Specific aspects of contemporary triathlon. Sports Med. 2002;32:1-15.

2. Lepers R. Analysis of Hawaii ironman performances in elite triathletes from 1981 to 2007. Med Sci Sports Exerc. 2008;40:1828-34.

3. International Triathlon Union. Olympic history. http://www.triathlon.org/olympics/history.

4. Knechtle B, Knechtle P, Lepers R. Participation and performance trends in ultratriathlons from 1985 to 2009. Scand J Med Sci Sports. 2011;e82-90.

5. Lenherr R, Knechtle B, Rüst CA, Rosemann T. Lepers R. From Double Iron to Double Deca Iron Ultra-Triathlon - A Retrospective Data Analysis from 1985 to 2011. Physical Sport Culture and Sport. Studies and Research. 2012;54:55-67.

6. Lepers R, Knetchle B, Knechtle P, Rosemann T. Analysis of ultra-triathlon Performances. Open Access Journal of Sports Medicine. 2011; 2:131-36.

7. Rust CA, Knechtle B, Knechtle P, Lepers R, Rosemann T, Onywera V. European athletes dominate performances in Double Iron ultra-triathlons - A retrospective data analysis from 1985 to 2010. European Journal of Sport Science, in press. http://dx.doi.org/10.1080/17461391.2011.641033.

8. Lepers R, Stapley P. Age-related changes in conventional road versus off-road triathlon performance. Eur J Appl Physiol. 2011;111(8):1687-94.

9. Lepers R. Stapley P. Differences in gender and performance in off-road triathlon. J Sports Sci. 2010; 28(14):1555-1562.

10. O'Toole ML, Douglas PS. Applied physiology of triathlon. Sports Med. 1995;19(4):251-67.

11. Hausswirth $C$, Lehénaff $D$. Physiological demands of running during long distance runs and triathlons. Sports Med. 2001;31(9):679-89.

12. Laursen PB, Rhodes EC. Factors affecting performance in an ultraendurance triathlon. Sports Med. 2001;31(3):195-209.

13. Millet GP, Dréano P, Bentley DJ. Physiological characteristics of elite short- and long-distance triathletes. Eur J Appl Physiol. 2003 Jan;88(4-5):427-30.

14. Millet GP, Bentley DJ. The physiological responses to running after cycling in elite junior and senior triathletes. Int J Sports Med. 2004 Apr;25(3):191-7.

15. Le Meur Y, Thierry B, Rabita G, Dorel S, Honnorat G, Brisswalter J, Hausswirth C. Spring-Mass Behaviour during the Run of an International Triathlon Competition. Int J Sports Med. 2013 Jan 17. [Epub ahead of print]

16. Millet GP, Vleck VE. Physiological and biomechanical adaptations to the cycle to run transition in Olympic triathlon: review and practical recommendations for training. $\mathrm{Br}$ J Sports Med. 2000 Oct;34(5):384-90. Review.

17. Millet GP, Millet GY, Hofmann MD, Candau RB. Alterations in running economy and mechanics after maximal cycling in triathletes: influence of performance level. Int J Sports Med. 2000 Feb;21(2):127-32.

18. O'Toole ML.Training for ultraendurance triathlons. Med Sci Sports Exerc. 1989 Oct;21:S209-13.

19. Laursen PB. Long distance triathlon: demands, preparation and performance. J Hum Sport Exerc. 2011; 6(2): 247-263.

20. Hausswirth C, Brisswalter J. Strategies for improving performance in long duration events: Olympic distance triathlon. Sports Med. 2008;38(11):881-91.

21. Jeukendrup AE, Jentjens RL, Moseley L. Nutritional considerations in triathlon. Sports Med. 2005;35(2):163-81. 
22. Dallam GM, Jonas S, Miller TK. Medical considerations in triathlon competition: recommendations for triathlon organizers, competitors and coaches. Sports Med. 2005;35(2):143-61.

23. Desgorces FD, Berthelot G, El Helou N, Thibault V, Guillaume M, Tafflet M, Hermine O, Toussaint JF. From Oxford to Hawaii ecophysiological barriers limit human progression in ten sport monuments. PLoS One. 2008;3(11):e3653.

24. Lepers R, Cattagni T. Do older athletes reach limits in their performance during marathon running? Age (Dordr). 2012;34:773-81.

25. Jokl P, Sethi PM, Cooper AJ. Master's performance in the New York City Marathon 1983-1999. Br J Sports Med. 2004;38(4):408-12.

26. Hunter SK, Stevens AA. Sex differences in marathon running with advanced age: physiology or participation? Med Sci Sports Exerc. 2013 Jan;45(1):148-56.

27. Lepers R, Rüst C, Stapley P, Knechtle B. Relative improvements in endurance performance with age: Evidence from 25 years of Hawaii Ironman racing. Age (Dordr), Epub 2012 fev 26. DOI: 10.1007/s11357-012-9392-z.

28. USA Triathlon. Triathlon participation, growth trends and demographics. Retrieved 13August, 2012, from http://www.usatriathlon.org/about-multisport/demographics.aspx.

29. Deaner RO. Distance running as an ideal domain for showing a sex difference in competitiveness. Arch Sex Behav. 2012 Jun 15. [Epub ahead of print]

30. Etter F, Knechtle B,Bukowski A, Rüst CA, Rosemann T, Lepers R. Age and gender interactions in short distance triathlon performance. J Sport Sci. in press.

31. Knechtle B, Rüst CA, Roseman T, Lepers R. Age- and gender-related differences in half-Ironman triathlon performances- the Ironman 70.3 Switzerland from 2007 to 2010. Open Access Journal of Sports Medicine. 2012;3:59-66.

32. Rüst CA, Knechtle B, Knechtle P, Rosemann T, Lepers R. Age of peak performance in elite male and female Ironman triathletes competing in a qualifier for 'Ironman Hawaii' - Ironman Switzerland from 1995-2011. Open Access Journal of Sports Medicine. 2012;3:175-82.

33. Rüst CA, Knechtle B, Knechtle P, Rosemann T, Lepers R. Participation and performance in Triple Iron ultra-triathlon - a cross-sectional and longitudinal data analysis. Asian J Sports Med. 2012;3:145-52.

34. Lepers R, Maffiuletti NA. Age and Gender Interactions in Ultra-Endurance Performance: Insight from Triathlon. Med Sci Sports Exerc. 2011;43:134-9.

35. Hoffman MD, Ong JC, Wang G. Historical Analysis of Participation in $161 \mathrm{~km}$ Ultramarathons in North America. Int J Hist Sport. 2010;27:1877-91.

36. Reaburn PRJ, Dascombe BJ, Janse de Jonge X. Body composition and gender differences in performance, in Nutritionnal Assesement of Athletes, Second Edition, Driskell JA, Wolinsky I , Eds CRC Press, Boca Raton, FL, 2011, 121-147.

37. Sparling PB.A meta-analysis of studies comparing maximal oxygen uptake in men and women. Res Q Exerc Sport. 1980;51(3):542-52.

38. Joyner MJ. Physiological limiting factors and distance running: influence of gender and age on record performances. Exerc Sport Sci Rev. 1993;21:103-33.

39. Bunc V, Heller J, Horcic J, Novotny J. Physiological profile of best Czech male and female young triathletes. J Sports Med Phys Fitness. 1996;36(4):265-70.

40. Shaskey DJ, Green GA. Sports haematology. Sports Med. 2000;29(1):27-38.

41. Wiswell RA, Hawkins SA, Jaque SV, Hyslop D, Constantino N, Tarpenning K, Marcell T, Schroeder ET. Relationship between physiological loss, performance decrement, and age in master athletes. J Gerontol A Biol Sci Med Sci.

2001;56(10):M618-26. 
42. Yasuda N, Gaskill SE, Ruby BC. No gender-specific differences in mechanical efficiency during arm or leg exercise relative to ventilatory threshold. Scand J Med Sci Sports. 2008;18(2):205-12.

43. Kimber NE, Ross JJ, Mason SL, Speedy DB. Energy balance during an ironman triathlon in male and female triathletes. Int J Sport Nutr Exerc Metab. 2002 Mar;12(1):47-62.

44. Landers GJ, Ong KB, Ackland TR, Blanksby BA, Main LC, Smith D. Kinanthropometric differences between 1997 World championship junior elite and 2011 national junior elite triathletes. J Sci Med Sport. 2012 Oct 19. doi:pii: S14402440(12)00194-6. 10.1016/j.jsams.2012.09.006.

45. Knechtle B, Wirth A, Baumann B, Knechtle P, Rosemann T, Oliver S. Differential correlations between anthropometry, training volume, and performance in male and female Ironman triathletes. J Strength Cond Res. 2010;24(10):2785-93.

46. Knechtle B, Wirth A, Baumann B, Knechtle P, Rosemann T. Personal best time, percent body fat, and training are differently associated with race time for male and female ironman triathletes. Res Q Exerc Sport. 2010;81(1):62-8.

47. VanHeest JL, Mahoney CE. Female athletes: factors impacting successful performance. Curr Sports Med Rep. 2007;6(3):190-4.

48. Zaryski, D.J. Smith, Training principles and issues for ultra-endurance athletes, Curr. Sports Med. Rep., 2005,4(3),165-70.

49. Cheuvront SN, Carter III R., DeRuisseau KC, Moffart RJ. Running performance differences between men and women. An update. Sports Med. 2005;35:1017-24.

50. Sparkling PB, O’Donnell EM, Snow TK. The gender difference in distance running performance has plateaued: an analysis of world rankings from 1980 to 1996 . Med Sci Sports Exerc. 1998;30:1725-29.

51. Speechly DP, Taylor SR, Rogers GG. Differences in ultraendurance exercise in performance-matched male and female runners. Med Sci Sports Exerc. 1996;28(3):359-65.

52. Hoffman MD. Ultramarathon trail running comparison of performance-matched men and women. Med Sci Sports Exerc. 2008;40(9):1681-6.

53. Whipp BJ, Ward SA. Will women soon outrun men? [Letter]. Nature 1992;335(6355):25.

54. Hoffman MD. Performance trends in 161-km ultramarathons. Int J Sports Med. 2010;31(1):31-7.

55. Hoffman MD, Wegelin JA. The Western States 100-Mile Endurance Run: participation and performance trends. Med Sci Sports Exerc. 2009;41(12):2191-8.

56. Rüst CA, Knechtle B, Knechtle P, Pfeifer S, Rosemann T, Lepers R et al. Gender difference and age-related changes in performance at the long distance duathlon World Championships. J Strength Cond Res. Epub 2012.

57. Rüst CA, Knechtle B, Rosemann T, Lepers R. Sex difference in race performance and age of peak performance in the Ironman Triathlon World Championship from 1983 to 2012. Extreme Physiology and Medicine, in press.

58. Sultana F, Brisswalter J, Lepers R, Hausswirth C, Bernard T. Effects of age and gender on Olympic triathlon performances. Sci Sport. 2008;23:130-35.

59. Tanaka H, Seals DR. Age and gender interactions in physiological functional capacity: insight from swimming performance. J Appl Physiol. 1997;82(3):846-51.

60. Eichenberger E, Knechtle B, Knechtle P, Rüst CA, Rosemann T, Lepers R. Best performances by men and women open-water swimmers during the 'English Channel Swim' from 1900 to 2010. J Sports Sci. 2012;30(12):1295-301. 
61. Eichenberger E, Knechtle B, Knechtle P, Rüst CA, Rosemann T, Lepers R. et al. Sex difference in open-water ultra-swim performance in the longest freshwater lake swim in Europe. J Strength Cond Res. Epub 2012 Jun 28.

62. Fischer G, Knechtle B, Rüst CA, Rosemann T. Male swimmers cross the English Channel faster than female swimmers. Scand J Med Sci Sports. Epub 2012 Nov 4.

63. Toussaint HM. Differences in propelling efficiency between competitive and triathlon swimmers. Med Sci Sports Exerc.1990;22(3):409-15.

64. Lavoie JM, Montpetit RR. Applied physiology of swimming. Sports Med. 1986;3:165-89.

65. McLean SP, Hinrichs RN. Sex differences in the centre of buoyancy location of competitive swimmers. J Sports Sci. 1998;16:373-83.

66. Pendergast DR, Di Prampero PE, Craig AB, Wilson DR, Rennie DW. Quantitative analysis of the front crawl in men and women. J Appl Physiol. 1977;43:475-79.

67. Pate RR, Sparling PB, Wilson GE, Cureton KJ, Miller BJ. Cardiorespiratory and metabolic responses to submaximal and maximal exercise in elite women distance runners. Int J Sports Med. 1987;8:91-5.

68. Zamparo P. Effects of age and gender on the propelling efficiency of the arm stroke. Eur J Appl Physiol. 2006 May;97(1):52-8.

69. Toussaint HM, de Groot G, Savelberg HH, Vervoorn K, Hollander AP, van Ingen Schenau GJ. Active drag related to velocity in male and female swimmers. J Biomech. 1988;21(5):435-8.

70. Schumacher YO, Mueller P, Keul J. Development of peak performance in track cycling. J Sports Med Phys Fitness. 2001;41:139-46.

71. Rüst CA, Knechtle B, Rosemann T, Lepers R. Men Cross America Faster than Women - the 'Race Across America' (RAAM) from 1982 to 2012. Int J Sports Physiol Perform. 2013 Feb 20. [Epub ahead of print]

72. Abou Shoak M, Knechtle B, Knechtle P, Rüst CA, Rosemann T, Lepers R.Participation and performance trends in ultra-cycling. Open Access Journal of Sports Medicine, 2013;4: 41-51.

73. Levis DA, Kamon E, Hodgson JL. Physiological differences between genders. Implications for sports conditioning. Sports Med. 1986;3:357-69.

74. Lucia A, Joyos H, Chicharro JL. Physiological response to professional road cycling: climbers vs time trialists. Int J Sports Med. 2000;21:505-12.

75. Impellizzeri FM, Marcora SM. The physiology of mountain biking. Sports Med. 2007;37(1):59-71.

76. Bernard T, Hausswirth C, Le Meur Y, Bignet F, Dorel S, Brisswalter J. Distribution of power output during the cycling stage of a triathlon world cup. Med Sci Sports Exerc. 2009;41(6), 1296-1302.

77. Le Meur Y, Hausswirth C, Dorel S, Bignet F, Brisswalter J, Bernard, T. Influence of gender on pacing adopted by elite triathletes during a competition. Eur J Appl Physiol. 2009;106(4), 535-45.

78. Hopkins WG, Schabort EJ, Hawley JA. Reliability of power in physical performance tests. Sports Med. 2001;31:211-34.

79. Stevenson J, Song H, Cooper JA. Age and Sex Differences Pertaining to Modes of Locomotion in Triathlon. Med Sci Sports Exerc. 2012 Dec 14. [Epub ahead of print]

80. Seiler S, D Koning JJ, Foster C. The fall and rise of the gender difference in elite anaerobic performance 1952-2006. Med Sci Sports Exerc. 2007;39:534-40.

81. Lepers R. Triathlon Science. Age and gender considerations in triathlon. In : Friel J editor. Human Kinetics, 2013:39-53. 
82. Mayhew JL, Salm PC. Gender differences in anaerobic power tests. Eur J Appl Physiol Occup Physiol. 1990;60(2):133-8.

83. Tanaka H, Seals DR. Endurance exercise performance in Masters athletes: ageassociated changes and underlying physiological mechanisms. J Physiol. 2008;586(1):55-63.

84. Ransdell LB, Vener J, Huberty J. Master athletes: An analysis of running, swimming and cycling performance by age and gender. J Exerc Sci Fit, 2009;7:S61-S73.

85. Tanaka H, Seals DR. Invited Review: Dynamic exercise performance in Masters athletes: insight into the effects of primary human aging on physiological functional capacity. J Appl Physiol. 2003;95(5):2152-62.

86. Maharam LG, Bauman PA, Kalman D, Skolnik H, Perle SM. Masters athletes: factors affecting performance. Sports Med. 1999;28:273-85.

87. Deaner RO. Physiology does not explain all sex differences in running performance. Med Sci Sports Exerc. 2013 Jan;45(1):146-7.

88. Reaburn P, Dascombe B. Endurance performance in masters athletes. Eur Rev Aging Phys Act. 2008;5:31-42.

89. Leyk D, Erley O, Ridder D, Leurs M, Rüther T, Wunderlich M, et al. Age-related changes in marathon and half-marathon performances. Int J Sports Med. 2007;28(6):513-7.

90. Knechtle B, Rüst CA, Rosemann T, Lepers R. Age-related changes in 100-km ultramarathon running performances. Age (Dordr), 2012 Aug;34(4):1033-45.

91. Lepers R, Stapley PJ, Cattagni T, Gremeaux V, Knechtle B. Limits in endurance performance of octogenarian athletes. J Appl Physiol. 2013 Mar;114(6):829.

92. Baker AB, Tang YQ. Aging performance for masters records in athletics, swimming, rowing, cycling, triathlon, and weightlifting. Exp Aging Res. 2010;36:453-77

93. Balmer J, Bird S, Davison R, Lucia A. Effect of age on 16.1-km time-trial performance. J Sports Sci. 2008 Jan 15;26(2):197-206.

94. Balmer J, Bird S, Davison R. Indoor 16.1-km time-trial performance in cyclists aged 25- 63 years. J Sports Sci. 2008 Jan 1;26(1):57-62.

95. Balmer J, Potter CR, Bird SR, Davison RC. Age-Related Changes in Maximal Power and Maximal Heart Rate Recorded During a Ramped Test in 114 Cyclists Age 15-73 Years. JAPA. 2005;13:125-36.

96. Bernard T, Sultana F, Lepers R, Hausswirth C, Brisswalter J. Age related decline in Olympic triathlon performance: effect of locomotion mode. Exp Aging Res. 2010;36:1-15

97. Knechtle B, Rüst CA, Knechtle P, Rosemann T, Lepers R. Age-related changes in ultra-triathlon performances. Extreme Physiology and Medicine, 2012 1:5

98. Lepers R, Sultana F, Bernard T, et al. Age-related changes in triathlon performances. Int J Sports Med 2010; 31(4):251-56.

99. Hunter SK, Stevens AA, Magennis K, Skelton KW, Fauth M. Is there a sex difference in the age of elite marathon runners? Med Sci Sports Exerc. 2011;43(4):656-64.

100. Kallinen M, Markku A. Aging, physical activity and sports injuries. An overview of common sports injuries in the elderly. Sports Med. 1995; 20:41-52.

101. Lepers R, Maffiuletti NA, Rochette L, Brugniaux J, Millet GY. Neuromuscular fatigue during a long-duration cycling exercise. J Appl Physiol. 2002;92:1487-93.

102. Bonnefoy M, Kostka T, Arsac LM, Berthouze SE, Lacour JR. Peak anaerobic power in elderly men. Eur J Appl Physiol. 1998;77:182-8.

103. Kostka T. Quadriceps maximal power and optimal shortening velocity in 335 men aged 23-88 years. Eur J Appl Physiol. 2005;95:140-55. 
104. McCrory JL, Salacinski AJ, Hunt SE, Greenspan SL. Thigh muscle strength in senior athletes and healthy controls. J Strength Cond Res. 2009;23:2430-36.

105. Stiefel M, Knechtle B, Lepers R. Master triathletes have not reached limits in their Ironman triathlon performance. Scand J Med Sci Sports. Epub 2012 May 14.

106. Medic N, Starkes JL, Young BW. Examining relative age effects on performance achievement and participation rates in Masters athletes. J Sports Sci. 2007;25(12):1377-84.

107. Gulbin JP, Gaffney PT. Ultraendurance triathlon participation: typical race preparation of lower level triathletes. J Sports Med Phys Fitness. 1999;39(1):12-5.

108. Knechtle B, Knechtle P, Rüst CA, Rosemann T. A comparison of anthropometric and training characteristics of Ironman triathletes and Triple Iron ultra-triathletes. J Sports Sci. 2011;29(13):1373-80.

109. Knechtle B, Wirth A, Rosemann T. Predictors of race time in male ironman triathletes: physical characteristics, training, or prerace experience? Percept Mot Skills. 2010;111(2):437-46.

110. Leyk D, Erley O, Gorges W, Ridder D, Rüther T, Wunderlich M et al. Performance, training and lifestyle parameters of marathon runners aged 20-80 years: results of the PACE-study. Int J Sports Med. 2009;30:360-65.

111. Wright VJ, Perricelli BC. Age-related rates of decline in performance among elite senior athletes. Am J Sports Med. 2008;36(3):443-50.

112. Gremeaux V, Gayda M, Lepers R, Sosner P, Juneau M, Nigam A. Exercise and longevity. Maturitas. 2012;73(4):312-17.

113. Trappe S, Hayes E, Galpin A, Kaminsky L, Jemiolo B, Fink W, Trappe T, Jansson A, Gustafsson T, Tesch P. New records in aerobic power among octogenarian lifelong endurance athletes. J Appl Physiol. 2013;114(1):3-10. 


\begin{tabular}{|c|c|c|c|c|}
\hline & Swim & Cycle & Run & Total \\
\hline \multicolumn{5}{|c|}{ Short Distance (1.5-40-10 km) : [30], [79] } \\
\hline \multicolumn{5}{|c|}{ Zurich (Switzerland) from 2000 to 2010} \\
\hline Top 5 elite overall & 15.2 & 13.4 & 17.1 & 14.8 \\
\hline Top 5 AG - from 18 to 54 years & 18.5 & 15.5 & 18.5 & 17.1 \\
\hline \multicolumn{5}{|c|}{ World Championship from 2009 to 2011} \\
\hline Top 10 AG - from 18 to 64 years & 13.3 & 10.7 & 7.5 & 12.0 \\
\hline \multicolumn{5}{|c|}{ Half Ironman (1.9- 90-21 km) : [31], [79] } \\
\hline \multicolumn{5}{|c|}{ Rapperswil (Switzerland) from 2007 to 2010} \\
\hline Top 5 elite overall & 14.1 & 12.3 & 12.5 & 12.6 \\
\hline Top 5 AG - from 18 to 54 years & 22.3 & 16.4 & 19.2 & 17.6 \\
\hline \multicolumn{5}{|c|}{ World Championship from 2009 to 2011} \\
\hline Top 10 AG - from 18 to 64 years & 12.4 & 11.2 & 14.5 & 12.6 \\
\hline \multicolumn{5}{|c|}{ Off-Road Triathlon (1.5-30-10 km) : [9] } \\
\hline \multicolumn{5}{|c|}{ World Championship (Maui, USA) from 2007 to 2009} \\
\hline Top 10 elite overall & 12.4 & 19.6 & 18.4 & 18.2 \\
\hline \multicolumn{5}{|c|}{ Ironman (3.8- 180-42 km) : [2], [32], [34] } \\
\hline \multicolumn{5}{|c|}{ World Championship (Kona, Hawaii, USA) from 1988 to 2007} \\
\hline Top 10 elite overall & 9.8 & 12.7 & 13.3 & 12.6 \\
\hline Top 10 AG - from 18 to 64 years & 12.1 & 15.4 & 18.2 & 15.8 \\
\hline \multicolumn{5}{|c|}{ Zurich (Switzerland) from 1995 to 2010} \\
\hline Top 10 elite overall & 14.0 & 13.2 & 18.2 & 14.9 \\
\hline
\end{tabular}

AG: Age Groups, [reference]

\section{Table 1}

Mean sex differences in time performance for swimming, cycling, running and total time at different national and international triathlons. 


\begin{tabular}{lccccccc} 
& \multicolumn{3}{c}{ Sex difference in performance } & \multicolumn{3}{c}{ Difference between } \\
Event & \multicolumn{2}{c}{ in top 10 athletes in 2012 (mean \pm SD) } & \multicolumn{2}{c}{ 10th and 1st (\%) } \\
Male & Female \\
\hline Hawaii Ironman Triathlon (3.8-180-42 km) & Swim & Cycle & Run & Total & \multicolumn{2}{c}{ Male } & Fund \\
Olympics Triathlon (1.5-40-10 km) with drafting & $14.1 \pm 7.9$ & $13.1 \pm 2.3$ & $7.3 \pm 2.9$ & $11.3 \pm 0.5$ & 3.6 & 4.0 \\
Hy-Vee Triathlon (1.5-40-10 km) without drafting & $8.6 \pm 4.8$ & $10.2 \pm 3.5$ & $8.6 \pm 4.4$ & $9.3 \pm 0.5$ & 4.1 & 3.3 \\
World Championship Off-Road Triathlon (1.5-30-10 km) & $15.2 \pm 15.5$ & $22.6 \pm 4.4$ & $15.1 \pm 6.7$ & $17.3 \pm 2.9$ & 4.6 & 12.2 \\
\hline
\end{tabular}

\section{Table 2}

Mean percentage differences in times for swimming, cycling, running and total event between the top 10 females and males and time difference between the winner and $10^{\text {th }}$ placed athlete in 2012 at four international triathlons: Hawaii Ironman triathlon World championship (Kona, Hawaii, USA). Olympics Triathlon (London, UK), Hy-Vee short distance triathlon (Des Moines, Iowa, USA); World championship off Road (Xterra) triathlon (Maui, Hawaii, USA). (Unpublished Personal data). 


\begin{tabular}{|c|c|c|c|c|c|c|c|c|c|c|}
\hline & \multicolumn{10}{|c|}{ Age Groups (years) } \\
\hline & $18-39$ & $40-44$ & $45-49$ & $50-54$ & $55-59$ & $60-64$ & $65-69$ & $70-74$ & $75-79$ & $>80$ \\
\hline \multicolumn{11}{|l|}{ Male } \\
\hline 3.8 km Swim (h:min:s) & $51: 56$ & $51: 48$ & $56: 55$ & 1:03:32 & 1:07:09 & 1:16:20 & 1:14:11 & $1: 47: 46$ & $1: 37: 47$ & 1:49:34 \\
\hline 180 km Cycle (h:min:s) & 4:24:05 & $4: 39: 16$ & 5:04:47 & $4: 51: 44$ & 5:00:17 & $5: 19: 17$ & $5: 42: 08$ & $5: 47: 33$ & 6:39:35 & 7:42:08 \\
\hline 42 km Run (h:min:s) & $2: 44: 02$ & $2: 53: 28$ & $3: 04: 21$ & $3: 24: 51$ & 3:34:03 & $3: 25: 28$ & $4: 14: 52$ & $3: 52: 47$ & $4: 55: 43$ & $5: 41: 51$ \\
\hline Total (h:min:s) & 8:03:56 & 8:24:32 & $9: 11: 24$ & $9: 26: 23$ & 9:47:29 & $10: 08: 15$ & $11: 19: 07$ & 11:45:05 & $13: 27: 50$ & $15: 38: 25$ \\
\hline $\begin{array}{l}\text { Total change (\%) } \\
\text { (year) }\end{array}$ & $\begin{array}{c}100 \\
(2011) \\
\end{array}$ & $\begin{array}{c}104 \\
(1994) \\
\end{array}$ & $\begin{array}{c}114 \\
(2009) \\
\end{array}$ & $\begin{array}{c}117 \\
(2006) \\
\end{array}$ & $\begin{array}{c}121 \\
(2005) \\
\end{array}$ & $\begin{array}{c}126 \\
(2010) \\
\end{array}$ & $\begin{array}{c}140 \\
(2011) \\
\end{array}$ & $\begin{array}{c}146 \\
(2011) \\
\end{array}$ & $\begin{array}{c}167 \\
(2005) \\
\end{array}$ & $\begin{array}{c}194 \\
(2012) \\
\end{array}$ \\
\hline \multicolumn{11}{|l|}{ Female } \\
\hline 3.8 km Swim (h:min:s) & $54: 31$ & 1:13:52 & 1:06:21 & 1:08:08 & 1:06:18 & 1:32:16 & 1:21:02 & $1: 37: 54$ & 1:45:05 & - \\
\hline 180 km Cycle (h:min:s) & 4:52:06 & $5: 25: 00$ & 5:06:07 & $5: 31: 56$ & $5: 35: 36$ & $6: 27: 46$ & $6: 47: 28$ & $7: 24: 33$ & $7: 25: 17$ & - \\
\hline 42 km Run (h:min:s) & 3:03:05 & $3: 17: 48$ & 3:09:18 & $3: 47: 23$ & 4:00:08 & 4:05:22 & 4:59:01 & $6: 07: 02$ & $6: 19: 43$ & - \\
\hline Total (h:min:s) & 8:54:02 & $10: 02: 35$ & $9: 26: 25$ & $10: 35: 59$ & $10: 51: 43$ & $12: 17: 24$ & $13: 16: 32$ & $15: 19: 19$ & $15: 54: 16$ & - \\
\hline $\begin{array}{l}\text { Total change (\%) } \\
\text { (year) }\end{array}$ & $\begin{array}{c}100 \\
(2009)\end{array}$ & $\begin{array}{c}113 \\
(2010)\end{array}$ & $\begin{array}{c}106 \\
(2012)\end{array}$ & $\begin{array}{c}119 \\
(2005)\end{array}$ & $\begin{array}{c}122 \\
(2010)\end{array}$ & $\begin{array}{c}138 \\
(2010)\end{array}$ & $\begin{array}{c}149 \\
(2010)\end{array}$ & $\begin{array}{c}172 \\
(2000)\end{array}$ & $\begin{array}{c}179 \\
(2005)\end{array}$ & \\
\hline
\end{tabular}

\section{Table 3}

Total time records (actualized with 2012 data) and corresponding split times for male and female age-groups at the Hawaii Ironman Triathlon between 1986 and 2012. The change in percentage compared to the fastest total time (age group 18-39 years) is given for each different age group. 